\section{Uso de la cinética del consumo de oxígeno para la evaluación de la capacidad cardiorrespiratoria en pacientes con obesidad}

\author{
CLAUDIA MIRANDA ${ }^{1, \mathrm{a}, \mathrm{b}}$, PAULINA IBACACHE ${ }^{1,2, \mathrm{a}, \mathrm{c}}$, \\ EDGARDO OPAZO ${ }^{3, \mathrm{a}, \mathrm{c}}$, JUAN ROJAS ${ }^{2, \mathrm{a}, \mathrm{d}, \mathrm{e}}$, MARCELO CANO $^{3, \mathrm{a}, \mathrm{f}}$
}

\section{Oxygen uptake kinetics to assess cardiorespiratory fitness in obese patients}

Background: The evaluation of cardiorespiratory fitness (RCC) using maximal or peak oxygen consumption $\left(\mathrm{VO}_{2}\right)$, requires a high level of effort in obese patients. We propose a method to evaluate RCC using constant and moderate loads, called $\mathrm{VO}_{2}$ kinetics (tau). Aim: To determine the relationship between tau and peak $\mathrm{VO}_{2}$ in patients with obesity. Material and Methods: Forty patients ( $87 \%$ females) aged $37 \pm 12$ years and with a body mass index (BMI) of $34.6 \pm$ $4.0 \mathrm{~kg} / \mathrm{m}^{2}$, were divided into two groups according to the applied workload ( 0.5 and 0.8 Watts $/ \mathrm{kg}$ body mass) using a cycle ergometer and Cortex Metalyzer $3 b$ equipment. The protocol was started with 6 minutes at constant load and then increments of 20-25 Watts every two min were made until determination of the peak $\mathrm{VO}_{2}$. Results: The tau value was $51.8 \pm 17.6 \mathrm{~s}$, the absolute peak $\mathrm{VO}_{2}$ was $2.0 \pm 0.7 \mathrm{~L} / \mathrm{min}$ and the relative peak $\mathrm{VO}_{2}$ was $26.6 \pm 30.0 \mathrm{ml} / \mathrm{kg} / \mathrm{min}$. There was a significant difference of tau medians between the group that used 0.5 and $0.8 \mathrm{Watts} / \mathrm{kg}(p=0.002)$ and a significant inverse correlation between the absolute peak $\mathrm{VO}_{2}$ and the tau value for a load of $0.5 \mathrm{Watts} / \mathrm{kg}$ ( rho $=-0.415$, $p=0.0327)$. Conclusions: The higher tau value, the lower the peak $\mathrm{VO}_{2}$ of an obese patient. It is suggested to apply loads of 0.5 Watts $/ \mathrm{kg}$ for a $\mathrm{VO}_{2}$ kinetics test in obese patients or in subjects who do not wish to carry out higher physiological demands with a non-invasive and low risk procedure.

(Rev Med Chile 2018; 146: 15-21)

Key words: Exercise Test; Obesity; Oxygen Consumption.
'Escuela de Kinesiología, Facultad de Ciencias de la Rehabilitación. Universidad Andres Bello. Santiago, Chile.

${ }^{2}$ Departamento de Morfología y Función, Universidad de las Américas, Viña del Mar, Chile. ${ }^{3}$ Departamento de Kinesiología, Universidad de Chile, Chile.

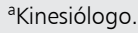

${ }^{\mathrm{b}}$ Magíster en Medicina y Ciencias del Deporte.

'Magíster en Fisiología.

${ }^{\mathrm{d}}$ Magíster en Ciencias Biológicas.

eMagíster en Educación.

${ }^{\mathrm{f}} \mathrm{PhD}$ en Fisiología del Ejercicio.

Recibido el 30 de mayo de 2017, aceptado el 2 de enero de 2018.

Correspondencia a:

Klga. Claudia Miranda F.

Escuela de Kinesiología,

Universidad Andres Bello.

Fernández Concha \#700, Edificio C5, Las Condes, Santiago, Chile. cmiranda@unab.cl

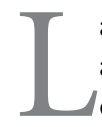

a obesidad se define como una acumulación anormal o excesiva de grasa, que lleva a un $\checkmark$ deterioro general en la salud de las personas por su alta asociación con otras patologías ${ }^{1-4}$. Según la Organización Mundial de la Salud (OMS), la prevalencia de obesidad en el mundo se ha incrementado de forma significativa ${ }^{5}$; en Chile, $39,3 \%$ de la población tiene sobrepeso y $25,1 \%$ obesidad $^{6}$, lo cual se atribuye a los hábitos de actividad física y el aumento de conductas sedentarias ${ }^{7-9}$.

La condición física es un conjunto de atributos físicos determinante de la capacidad de realizar un trabajo pudiendo o no estar relacionado con la salud $^{10,11}$; la capacidad cardiorrespiratoria (CCR) es un componente de esta condición utilizado como indicador de salud y de expectativa de vida, siendo objetivada a través del consumo de oxígeno máximo $\left(\mathrm{VO}_{2} \max \right)^{12-14}$; corresponde a la mayor cantidad de oxígeno que una persona puede consumir, transportar y utilizar en una inspiración durante la realización de un ejercicio dinámico ${ }^{15,16}$. Para evaluar esta variable se necesita 
de las máximas capacidades cardiorrespiratorias del sujeto ${ }^{17,18}$, situación que puede resultar riesgosa en algunos $\operatorname{casos}^{19}$. En pacientes con obesidad, estas evaluaciones generalmente son submáximas y se utiliza el término consumo de oxígeno peak $\left(\mathrm{VO}_{2}\right.$ peak) para denominar la cantidad de oxígeno consumido en el momento de la detención de la prueba $^{15,20-22}$, el cual se expresa en valores absolutos $(\mathrm{L} / \mathrm{min})$ o relativos al peso corporal $(\mathrm{ml} / \mathrm{kg} / \mathrm{min})^{23}$. Uno de los aspectos poco descritos del $\mathrm{VO}_{2}$ es su cinética, la cual consiste en la respuesta dinámica del $\mathrm{VO}_{2}$ al comienzo de un ejercicio de carga constante durante 6 a 10 minutos, aproximadamente $^{24-26}$. Para poder describir el comportamiento de la cinética del $\mathrm{VO}_{2}$ a carga subumbral, se utiliza la expresión matemática: $\mathrm{VO}_{2}=\mathrm{BL}+\mathrm{A}\left(1-\mathrm{e}^{-(\mathrm{t}-\mathrm{TD} / \tau)}\right)$, donde $\mathrm{VO} 2$ = consumo de oxígeno en función del tiempo; $\mathrm{BL}=$ consumo de oxígeno inicial o de base; $\mathrm{A}=$ ganancia total del consumo de oxígeno; $\mathrm{e}=$ base del logaritmo natural, $\mathrm{t}=$ tiempo; $\tau=$ tau; $\mathrm{TD}=$ tiempo de retraso que precede al incremento en el $\mathrm{VO}_{2}$ muscular ${ }^{27}$.

En la respuesta de la cinética del $\mathrm{VO}_{2}$ durante ejercicio a carga constante se reconocen 3 fases (Figura 1). La primera de ellas se relaciona con el aumento del gasto cardíaco; la segunda responde a un aumento exponencial del $\mathrm{VO}_{2}$ con un valor constante de tiempo tau $(\tau)$ y tiene relación con la llegada de sangre venosa proveniente de los músculos en ejercicio, representando la capacidad de utilizar el oxígeno. Finalmente, la fase III presenta un estado estable, siendo posible obser- varla cuando el ejercicio se realiza bajo el umbral ventilatorio ${ }^{28}$.

La fase II y, específicamente el valor de $\tau$, que corresponde al tiempo en segundos (s) donde se obtiene el 63\% de la amplitud máxima de la meseta del consumo de oxígeno con carga submáxima, ha demostrado ser sensible a cambios de la CCR en pacientes con patologías cardiorrespiratorias ${ }^{29}$ y diabetes mellitus tipo $\mathrm{II}^{30}$, asociándose la disminución del valor de $\tau$ a una mejor $\mathrm{CCR}^{31}$. Se ha demostrado que la respuesta de esta variable es dependiente de la intensidad y solo tiene un comportamiento monoexponencial con cargas moderadas de trabajo; si el ejercicio es intenso, se pierde la estabilidad del $\mathrm{VO}_{2}$, aumentando de manera constante en el tiempo, lo que se conoce como componente lento de la cinética del $\mathrm{VO}_{2}^{24,32,33}$.

Actualmente, existe escasa literatura del comportamiento de $\tau$ en sujetos con obesidad ${ }^{34}$. El objetivo del presente trabajo es determinar la carga de trabajo óptimo para la aplicación de la prueba de cinética del consumo de oxígeno en sujetos con obesidad y establecer su relación con el consumo de oxígeno peak en este tipo de pacientes.

\section{Material y Método}

\section{Diseño y participantes}

El presente es un estudio descriptivo y correlacional, realizado durante los años 2015 y 2016 en el laboratorio de Fisiología del Ejercicio de la

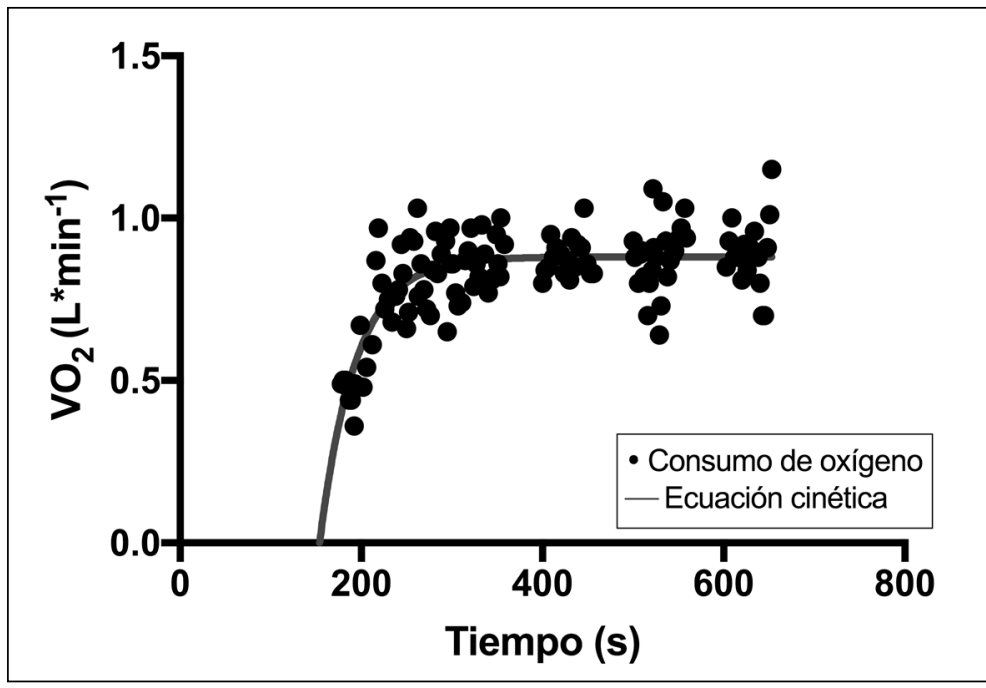

Figura 1. Ilustración esquemática de la respuesta del consumo de $\mathrm{O}_{2}$ de un sujeto durante una prueba a carga estable moderada en cicloergómetro. (Elaboración propia). 
carrera de Kinesiología de la Universidad Andres Bello en la Quinta Región de Chile; cumplió con las pautas señaladas en la Declaración de Helsinki 2013 y aprobado por el Comité de Ética en Investigación en Seres Humanos de la Facultad de Medicina de la Universidad de Chile, Proy. $\mathrm{N}^{\circ}$ 149-2014; todos los participantes firmaron su consentimiento informado previo a la toma de datos. La muestra fue obtenida por conveniencia, alcanzando un total de 40 participantes de acuerdo a los siguientes criterios de inclusión: IMC $\geq 30$ $\mathrm{kg} / \mathrm{m}^{2}$, haber cumplido con los criterios quirúrgicos y anestésicos para efectuarse una cirugía bariátrica y que la intervención indicada sea una gastrectomía vertical. Se excluyeron todos aquellos pacientes con cirugía bariátrica previa, portadores de comorbilidades médicas como: enfermedad respiratoria crónica, cardíaca, hepática crónica o insuficiencia renal, pacientes que utilizaran beta bloqueadores, con hábito tabáquico, mujeres postmenopáusicas y pacientes que presentaran patología musculoesquelética que impidiera la realización de las pruebas.

\section{Procedimientos}

Cada sujeto debió cumplir un ayuno de $4 \mathrm{~h}$ previas y no efectuar actividad física intensa en las $24 \mathrm{~h}$ antes de la evaluación. Para la obtención de los datos se utilizó un equipo Metalyzer $3 b$, Cortex (CORTEX Biophysik, Leipzig, Alemania) y un cicloergómetro Monark 915E (Monark Exercise $\mathrm{AB}$, Vansbro, Suecia). La muestra se dividió en dos grupos, a uno de ellos se aplicó una carga de trabajo de 0,5 watts por kilogramo de masa corpo$\mathrm{ral}(\mathrm{W} / \mathrm{kg})(\mathrm{n}=26)$ y al otro una carga de $0,8(\mathrm{~W} /$ $\mathrm{kg})(\mathrm{n}=14)$, lo anterior con el fin de encontrar la carga adecuada para la estabilización del consumo de oxígeno. El protocolo consistió en 2 minutos de medición de parámetros basales cardiorrespiratorios en reposo sobre el cicloergómetro, para luego pedalear con una cadencia de $60 \mathrm{rpm}$ a carga constante durante 6 minutos. Posterior a esto, para conseguir los valores de $\mathrm{VO}_{2}$ peak, se incrementó la carga en escalones de 20-25 W cada dos minutos hasta llegar a la fatiga o alcanzar un cociente respiratorio $(\mathrm{RER}) \geq 1,1$, una percepción de esfuerzo $\geq 7$ en escala de Borg modificada o que el sujeto manifestara fatiga muscular que le impidiera mantener la cadencia de pedaleo de $60 \mathrm{rpm}^{36}$. Posterior a esto se mantuvo un pedaleo sin carga durante 3 minutos a modo de vuelta al reposo. Para definir $\tau$ se empleó el protocolo descrito por Poole ${ }^{25}$, donde el valor $\tau$ se obtuvo de los 6 minutos iniciales de la prueba de ejercicio en cicloergómetro. Los valores de consumo de oxígeno ventilación por ventilación fueron transformados para obtener valores con la frecuencia de $1 \mathrm{~Hz}$, para ser modelados matemáticamente según la ecuación que define la respuesta del $\mathrm{VO}_{2}$ a carga constante e intensidades moderadas antes mencionada.

\section{Análisis estadístico}

Se comprobó la normalidad de las variables: carga inicial (W), consumo oxígeno peak absoluto $(\mathrm{L} / \mathrm{min})$, consumo oxígeno peak relativo $(\mathrm{ml} / \mathrm{kg} /$ min) y tau (s), a través del test de normalidad de Skapiro-Wilk. Para la comparación de medianas se utilizó la prueba de Wilcoxon y para la asociación de variables la prueba estadística de Spearman. Para los resultados fue aceptado un nivel de significancia del 95\% $(\mathrm{p}<0,05)$. Para los análisis estadísticos se utilizó el programa Statistics/Data Analysis Stata ${ }^{\circledR} 11.0$.

\section{Resultados}

De 40 sujetos evaluados, $87 \%$ correspondió a mujeres y $13 \%$ a hombres. Esta muestra fue dividida en dos grupos, según carga de trabajo aplicada, $0,5 \mathrm{~W} / \mathrm{kg}$ y $0,8 \mathrm{~W} / \mathrm{kg}$; las características de los participantes se muestran en la Tabla 1. El grupo que utilizó carga de 0,5 W/kg presentó un valor promedio de $\tau$ de $44,3 \pm 12,9 \mathrm{~s}$, un $\mathrm{VO}_{2}$ peak absoluto de $2,1 \pm 0,6 \mathrm{~L} / \mathrm{min}$ y un $\mathrm{VO}_{2}$ peak relativo de $21,6 \pm 3,6 \mathrm{ml} / \mathrm{kg} / \mathrm{min}$, a diferencia del grupo que utilizó una carga del $0,8 \mathrm{~W} / \mathrm{kg}$, el cual presentó un valor promedio de $\tau$ de $66,9 \pm 16,9 \mathrm{~s}$, un $\mathrm{VO}_{2}$ peak absoluto de $1,9 \pm 0,7 \mathrm{~L} /$ min y un $\mathrm{VO}_{2}$ peak relativo de $22,4 \pm 6,3 \mathrm{ml} / \mathrm{kg} / \mathrm{min}$.

$\mathrm{Al}$ estudiar el tipo de distribución de datos,

Tabla 1. Características de los pacientes incluidos en el estudio

\begin{tabular}{|lcc|}
\hline Variable & Carga 0,5 W/kg & Carga 0,8 W/kg \\
\hline Edad (años) & $34,3 \pm 10,6$ & $42,5 \pm 11,8$ \\
\hline Peso $(\mathrm{kg})$ & $94,5 \pm 16,0$ & $87,1 \pm 16,5$ \\
\hline Talla $(\mathrm{m})$ & $1,63 \pm 0,1$ & $1,6 \pm 0,11$ \\
\hline IMC $\left(\mathrm{kg} / \mathrm{m}^{2}\right)$ & $35,5 \pm 3,7$ & $32,8 \pm 4,5$ \\
\hline
\end{tabular}

Datos presentados como media \pm desviación estándar. 


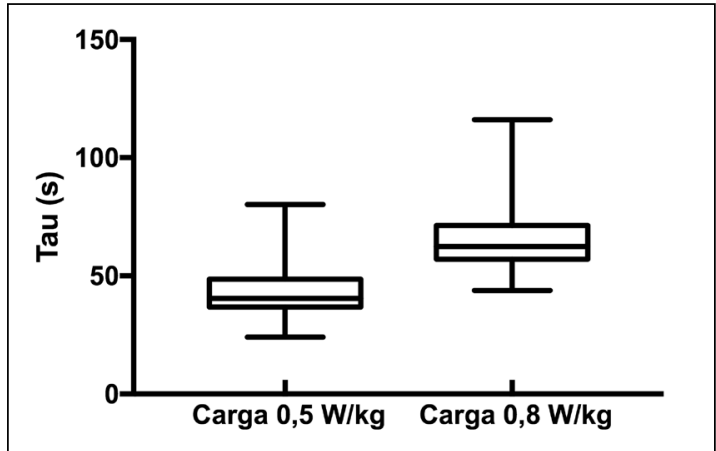

Figura 2. Diferencia para las medianas de tau (s) según carga de trabajo $(p=0,002)$.
Tabla 2. Resultados de $\mathrm{VO}_{2}$ peak y cinética del consumo de oxígeno según carga de trabajo

\begin{tabular}{|lccc|}
\hline Carga & $\begin{array}{c}\text { Carga } \\
\mathbf{0 , 5} \mathbf{~ W / k g}\end{array}$ & $\begin{array}{c}\text { Carga } \\
\mathbf{0 , 8} \mathbf{~ W / k g}\end{array}$ & $\begin{array}{c}\mathbf{p} \\
\text { valor }\end{array}$ \\
$\begin{array}{l}\text { VO } \\
\text { absoluto } \text { peak }\end{array}$ & $2,1 \pm 0,6$ & $1,9 \pm 0,7$ & $\mathrm{p}=0,2068$ \\
$\begin{array}{l}\text { VO }{ }_{2} \text { peak relativo } \\
\left(\mathrm{ml}^{\prime} \mathrm{kg}^{-1} / \mathrm{min}^{-1}\right)\end{array}$ & $21,6 \pm 3,6$ & $22,4 \pm 6,3$ & $\mathrm{p}=0,9322$ \\
tau (s) & $44,3 \pm 12,9$ & $66,9 \pm 16,9 \mathbf{p}=\mathbf{0 , 0 0 0 2}$ \\
\hline
\end{tabular}

Datos presentados como media \pm desviación estándar. Nivel de significancia $(p<0,05)$ para la diferencia de media de la respectiva variable.

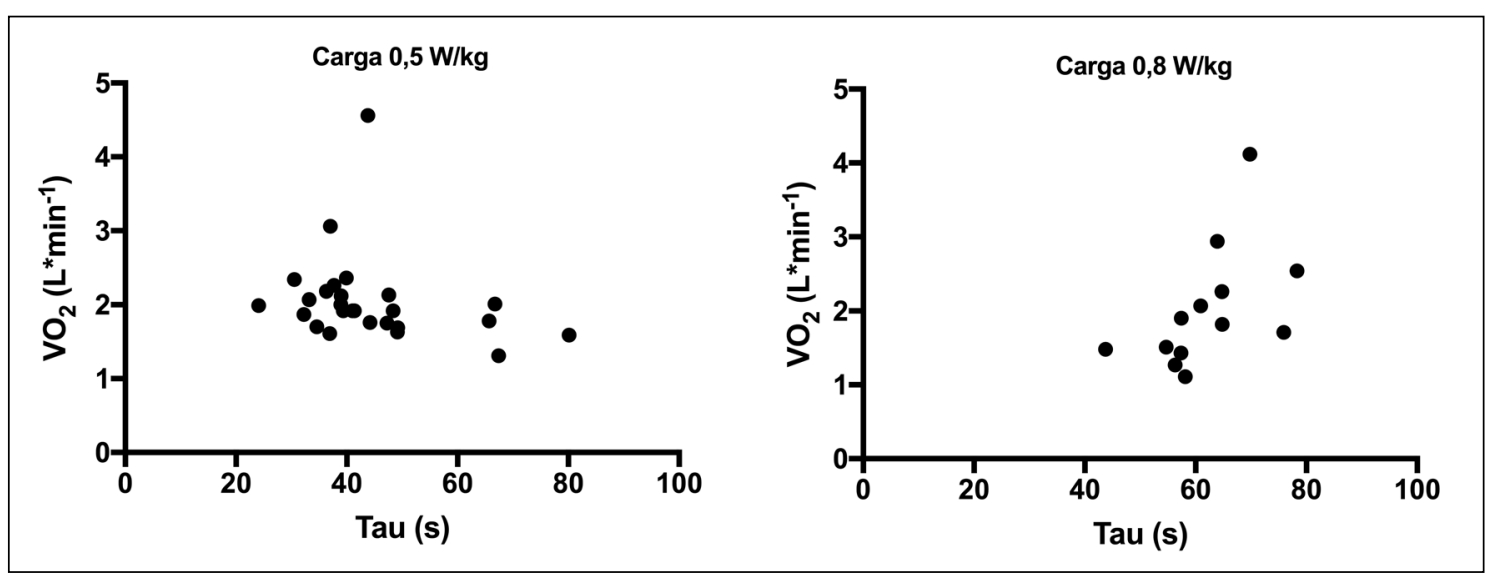

Figura 3. Relación entre consumo de oxígeno peak absoluto (l/min) y tau (s) según carga de trabajo aplicada (W/kg).

tanto la variable $\tau(\mathrm{p}=0,00342)$, como la variable $\mathrm{VO}_{2}$ peak absoluto $(\mathrm{p}=0,000)$ y $\mathrm{VO}_{2}$ peak relativo $(\mathrm{p}=0,000)$ no presentaron distribución normal.

Se encontraron diferencias estadísticas significativas entre las medianas de $\tau(\mathrm{p}=0,0002)$ para las cargas de $0,5 \mathrm{~W} / \mathrm{kg}$ y $0,8 \mathrm{~W} / \mathrm{kg}$, no existiendo diferencias significativas para el $\mathrm{VO}_{2}$ peak relativo $(\mathrm{p}=0,9322)$ ni para el $\mathrm{VO}_{2}$ peak absoluto $(\mathrm{p}=$ 0,2068 ) al comparar ambos grupos (Figura 2).

La relación entre las variables de $\tau$ y el $\mathrm{VO}_{2}$ peak (absoluto y relativo) también fue analizada según la influencia de la carga de trabajo aplicada. Se observó una relación inversa significativa entre el $\mathrm{VO}_{2}$ peak absoluto y $\tau$ en el grupo que utilizó la carga de $0,5 \mathrm{~W} / \mathrm{kg}($ rho $=-0,4199 ; \mathrm{p}=0,0327)$ (Figura 3). Por el contrario, la relación entre $\tau$ y $\mathrm{VO}_{2}$ peak relativo para la carga de $0,5 \mathrm{~W} / \mathrm{k}$ no resultó significativa ( $r$ ho $=0,2923 ; \mathrm{p}=0,3105$ ). Para los análisis de la carga de trabajo de $0,8 \mathrm{~W} /$ $\mathrm{kg}$, la relación resultó no significativa entre $\tau \mathrm{y}$ $\mathrm{VO}_{2}$ peak absoluto (rho $=-0,3242 ; \mathrm{p}=0,1062$ ) y entre $\tau$ y $\mathrm{VO}_{2}$ peak relativo $($ rho $=0,0769 ; \mathrm{p}=$ 0,7938 ) (Tabla 2).

\section{Discusión}

La caracterización de la cinética del consumo de oxígeno proporciona información valiosa con respecto a la efectividad del acoplamiento de la liberación de $\mathrm{O}_{2}$ a las demandas metabólicas de los tejidos y la capacidad del propio músculo de 
utilizar este $\mathrm{O}_{2}$ para la fosforilación oxidativa ${ }^{35}$. Se ha descrito que los menores valores $(\tau \sim 10 \mathrm{~s})$ se han encontrado en individuos con alto nivel de entrenamiento físico ${ }^{32}$ y entre 20 y 60 segundos en individuos adultos $\operatorname{sanos}^{33}$; lo anterior contrasta con los valores promedios obtenidos de $\tau$ en esta investigación para sujetos con obesidad $(44,30$ segundos a $0,5 \mathrm{~W} / \mathrm{kg}$ y 65,86 segundos a $0,8 \mathrm{~W} /$ $\mathrm{kg}$ ), lo que puede indicar una retrasada capacidad de respuesta del sistema cardiorrespiratorio a la carga de trabajo ${ }^{36}$. En sujetos sanos, el aumento de la captación de oxígeno al inicio del ejercicio refleja los ajustes circulatorios a los cambios metabólicos inducidos por esta actividad; en ellos, el aporte de oxígeno no es limitante durante el ejercicio submáximo, lo que refleja principalmente la eficiente bioenergética muscular y difusión por parte del sistema cardiorrespiratorio del oxígeno a nivel tisular ${ }^{37}$, condición que se ha demostrado deteriorada en los sujetos con obesidad ${ }^{36}$.

De los valores de $\tau$ obtenidos en esta investigación se desprende que, entre mayor sea la carga de trabajo de una prueba de esfuerzo $(0,8 \mathrm{~W} / \mathrm{kg})$, el valor de $\tau$ tiende a ser mayor, lo que concuerda con los resultados obtenidos por Robergs ${ }^{38}$. Para comprender mejor este resultado debemos partir de la base que sujetos obesos presentan capacidades cardiorrespiratorias significativamente inferiores a los de sujetos eutróficos ${ }^{36}$; además, estos, al realizar ejercicio físico, disminuyen su volumen corriente y la ventilación por minuto, incrementando la frecuencia respiratoria para compensar la reducción del volumen corriente; esta condición estimula la aparición del umbral ventilatorio, acelerando el inicio del componente lento de la cinética del $\mathrm{VO}_{2}$, perjudicando la tolerancia al esfuerzo y la adaptación al ejercicio ${ }^{22,36}$.

Recientemente ha sido publicado un estudio que describe el comportamiento de $\tau$ en pacientes adultos con obesidad ${ }^{34}(58,73 \pm 35,82 \mathrm{~s})$ y en adolescentes en la misma condición $(34,5 \pm 7,5$ s) ${ }^{39}$. Por otro lado, también se han encontrado datos del comportamiento de esta variable en pacientes con enfermedad vascular pulmonar (74 $\pm 16 s)^{40} y$ diabetes mellitus II $(55,7 \pm 20,6 s)^{41}$. En las publicaciones mencionadas anteriormente, se atribuye el aumento del valor de $\tau$ a una variedad de factores, incluyendo la intensidad de la carga de trabajo y la capacidad del sistema cardiovascular para responder a la mayor demanda de ejercicio. Por otra parte, Simoneau y Kelley dejaron en evidencia que existe una reducida actividad de las enzimas oxidativas y un aumento desproporcionado de la actividad de las enzimas glucolíticas en sujetos con exceso de peso tanto en aquellos con DM II como en aquellos sin esta condición ${ }^{42}$, lo que podría explicar los resultados de $\tau$ obtenidos en esta investigación.

Respecto a los datos de $\mathrm{VO}_{2}$ peak relativo obtenidos, estos se encuentran muy por debajo del punto de corte planteado por Blair en hombres y mujeres para tener un menor riesgo de mortalidad por todas las causas $(31,5$ y $35,4 \mathrm{ml} / \mathrm{kg} /$ min respectivamente) (Tabla 1$)^{43}$. En adultos, la variable $\tau$ suele estar inversamente relacionada con el consumo máximo de oxígeno ${ }^{33}$; es así como en esta investigación, $\tau$ está relacionado inversamente con el $\mathrm{VO}_{2}$ peak absoluto al utilizar cargas de 0,5 $\mathrm{W} / \mathrm{kg}$, no encontrándose una relación con cargas de $0,8 \mathrm{~W} / \mathrm{kg}$, evidenciando que a un mayor valor de $\tau$ menor es el resultado del $\mathrm{VO}_{2}$ peak absoluto de un sujeto con obesidad.

Estos resultados sugieren la utilidad y aplicabilidad de la prueba submáxima a carga constante $(\tau)$ como una importante herramienta de evaluación de la CCR en sujetos con obesidad, en los cuales se prefiere evitar la realización de una evaluación de $\mathrm{VO}_{2}$ max o peak, por los riesgos que estas conllevan en sujetos que suelen poseer distintos factores de riesgo cardiovasculares asociados.

A pesar de la significancia estadística y clínica de estos resultados, la correlación obtenida se describe como débil ${ }^{44}$, lo que puede ser consecuencia del tamaño muestral de cada grupo evaluado. En consecuencia, se sugiere realizar más estudios al respecto que consideren esta recomendación, de tal manera de potenciar los resultados de la presente investigación y la utilidad de esta variable en la población con obesidad.

\section{Conclusión}

Se concluye que existen diferencias en los valores de $\tau$ en pacientes con obesidad de acuerdo a las cargas de trabajo utilizadas. Aplicando la carga adecuada $(0,5 \mathrm{~W} / \mathrm{kg})$, el valor de $\tau$ resulta ser un buen indicador de la capacidad cardiorrespiratoria del sujeto con obesidad por su correlación con el $\mathrm{VO}_{2}$ peak absoluto.

En consecuencia, se sugiere aplicar cargas de $0,5 \mathrm{~W}$ por kilogramo de masa corporal para una 
prueba de cinética del $\mathrm{VO}_{2}$ en pacientes con obesidad o en sujetos que no se desee llevar a mayores demandas fisiológicas, siendo esta un procedimiento de bajo riesgo y compromiso fisiológico, no invasivo y muy bien tolerado por los pacientes.

Agradecimientos: Se agradece de manera especial a la Universidad Andres Bello, Facultad de Ciencias de la Rehabilitación, carrera de Kinesiología por permitir realizar este trabajo.

\section{Referencias}

1. Poobalan A, Aucott L. Obesity Among Young Adults in Developing Countries: A Systematic Overview. Currt Obes Rep 2016; 5: 2-13.

2. Carlsson AC, Arnlov J, Sundstrom J, Michaelsson K, Byberg L, Lind L. Physical activity, obesity and risk of cardiovascular disease in middle-aged men during a median of 30 years of follow-up. Eur J Prev Cardiol 2016; 23 (4): 359-65.

3. Popkin BM, Adair LS, Ng SW. Global nutrition transition and the pandemic of obesity in developing countries. Nutrition Reviews 2012; 70 (1): 3-21.

4. WHO. Obesidad y sobrepeso 2016 [cited 2016. Available from: http://www.who.int/mediacentre/factsheets/fs311/ es/.

5. Ng M, Fleming T, Robinson M, Thomson B, Graetz N, Margono C, et al. Global, regional, and national prevalence of overweight and obesity in children and adults during 1980-2013: a systematic analysis for the Global Burden of Disease Study 2013. Lancet 2014; 384 (9945): 766-81.

6. MINSAL. Encuesta Nacional de Salud 2009-2010 2009-2010 [cited 2016 diciembre 13]. Available from: http://web.minsal.cl/portal/url/item/bcb03d7bc28b64dfe040010165012d23.pdf.

7. Popkin BM, Gordon-Larsen P. The nutrition transition: worldwide obesity dynamics and their determinants. Int J Obes Relat Metab Disord 2004; 28 Suppl 3: S2-9.

8. Tudor-Locke C, Brashear MM, Johnson WD, Katzmarzyk PT. Accelerometer profiles of physical activity and inactivity in normal weight, overweight, and obese U.S. men and women. Int J Behav Nutr Phys Act 2010; 7: 60.

9. Van Dyck D, Cerin E, De Bourdeaudhuij I, Hinckson E, Reis RS, Davey R, et al. International study of objectively measured physical activity and sedentary time with body mass index and obesity: IPEN adult study. Int J Obes 2015; 39 (2): 199-207.

10. Caspersen CJ, Powell KE, Christenson GM. Physical activity, exercise, and physical fitness: definitions and distinctions for health-related research. Public Health Reports 1985; 100 (2): 126-31.

11. Goto Y, Yokokawa H, Fukuda H, Naito T, Hisaoka T, Isonuma $\mathrm{H}$. Body mass index and waist circumference are independent risk factors for low vital capacity among Japanese participants of a health checkup: a single-institution cross-sectional study. Environ Health Prev Med 2015; 20 (2): 108-15.

12. Myers J, Prakash M, Froelicher V, Do D, Partington S, Atwood JE. Exercise Capacity and Mortality among Men Referred for Exercise Testing. N Engl J Med 2002; 346 (11): 793-801.

13. Kurl S, Laukkanen JA, Rauramaa R, Lakka TA, Sivenius J, Salonen JT. Cardiorespiratory fitness and the risk for stroke in men. Arch Internal Med 2003; 163 (14): 16828.

14. Gander J, Lee D-c, Sui X, Hébert JR, Hooker SP, Blair $\mathrm{SN}$. Self-rated health status and cardiorespiratory fitness as predictors of mortality in men. Brit J Sports Med 2011; 45 (14): 1095-100.

15. ATS/ACCP Statement on cardiopulmonary exercise testing. Am J Respir Critical Care Med 2003; 167 (2): 211-77.

16. Arena R, Myers J, Guazzi M. The future of aerobic exercise testing in clinical practice: is it the ultimate vital sign? Future Cardiol 2010; 6 (3): 325-42.

17. Bentley DJ, Newell J, Bishop D. Incremental exercise test design and analysis: implications for performance diagnostics in endurance athletes. Sports Medicine (Auckland, NZ) 2007; 37 (7): 575-86.

18. Midgley AW, McNaughton LR, Polman R, Marchant D. Criteria for determination of maximal oxygen uptake: a brief critique and recommendations for future research. Sports Med (Auckland, NZ) 2007; 37 (12): 1019-28.

19. Mezzani A, Agostoni P, Cohen-Solal A, Corra U, Jegier A, Kouidi E, et al. Standards for the use of cardiopulmonary exercise testing for the functional evaluation of cardiac patients: a report from the Exercise Physiology Section of the European Association for Cardiovascular Prevention and Rehabilitation. Eur J Cardiovasc Prev Rehabil 2009; 16 (3): 249-67.

20. Noonan V, Dean E. Submaximal exercise testing: clinical application and interpretation. Phys Therapy 2000; 80 (8): 782-807.

21. Forman DE, Myers J, Lavie CJ, Guazzi M, Celli B, Arena R. Cardiopulmonary exercise testing: relevant but underused. Postgrad Med 2010; 122 (6): 68-86.

22. Arena R, Cahalin LP. Evaluation of cardiorespiratory fitness and respiratory muscle function in the obese population. Prog Cardiovas Dis 2014; 56 (4): 457-64. 
23. Fletcher GF, Ades PA, Kligfield P, Arena R, Balady GJ, Bittner VA, et al. Exercise standards for testing and training: a scientific statement from the American Heart Association. Circulation 2013; 128 (8): 873-934.

24. Grassi B. Oxygen uptake kinetics: old and recent lessons from experiments on isolated muscle in situ. Eur J Appl Physiol 2003; 90 (3-4): 242-9.

25. Poole DC, Jones AM. Oxygen uptake kinetics. Compr Physiol 2012; 2 (2): 933-96.

26. Whipp BJ, Wasserman K. Oxygen uptake kinetics for various intensities of constant-load work. J Appl Physiol 1972; 33 (3): 351-6.

27. Jones AM, Burnley M. Oxygen uptake kinetics: an underappreciated determinant of exercise performance. Int J Sports Physiol Perform 2009; 4 (4): 524-32.

28. Grassi B, Poole DC, Richardson RS, Knight DR, Erickson BK, Wagner PD. Muscle O2 uptake kinetics in humans: implications for metabolic control. J Appl Physiol (Bethesda, Md: 1985) 1996; 80 (3): 988-98.

29. Borghi-Silva A, Beltrame T, Reis MS, Sampaio LM, Catai $\mathrm{AM}$, Arena R, et al. Relationship between oxygen consumption kinetics and BODE Index in COPD patients. Int J Chron Obstruct Pulmon Dis 2012; 7: 711-8.

30. Brandenburg SL, Reusch JE, Bauer TA, Jeffers BW, Hiatt WR, Regensteiner JG. Effects of exercise training on oxygen uptake kinetic responses in women with type 2 diabetes. Diabetes Care 1999; 22 (10): 1640-6.

31. Franco RL, Bowen MK, Arena R, Privett SH, Acevedo EO, Wickham EP, et al. Sex differences in pulmonary oxygen uptake kinetics in obese adolescents. J Pediatr 2014; 165 (6): 1161-5.

32. Koppo K, Bouckaert J, Jones AM. Effects of training status and exercise intensity on phase II $\mathrm{VO}_{2}$ kinetics. Med Sci Sports Exerc 2004; 36 (2): 225-32.

33. Whipp BJ, Rossiter HB, Ward SA. Exertional oxygen uptake kinetics: a stamen of stamina? Biochem Soc Trans 2002; 30 (2): 237-47.

34. Neunhaeuserer D, Gasperetti A, Savalla F, Gobbo S, Bullo V, Bergamin M, et al. Functional Evaluation in
Obese Patients Before and After Sleeve Gastrectomy. Obes Surg 2017.

35. Whipp BJ, Ward SA, Rossiter HB. Pulmonary O2 uptake during exercise: conflating muscular and cardiovascular responses. Med Sci Sports Exerc 2005; 37 (9): 1574-85.

36. Littleton SW. Impact of obesity on respiratory function. Respirology (Carlton, Vic) 2012; 17 (1): 43-9.

37. Ba A, Bregeon F, Delliaux S, Cisse F, Samb A, Jammes Y. Cardiopulmonary response to exercise in COPD and overweight patients: relationship between unloaded cycling and maximal oxygen uptake profiles. Biomed Res Int 2015; 2015: 378469.

38. Robergs RA. A critical review of the history of low- to moderate-intensity steady-state VO2 kinetics. Sports Med (Auckland, NZ) 2014; 44 (5): 641-53.

39. Salvadego D, Lazzer S, Busti C, Galli R, Agosti F, Lafortuna $\mathrm{C}$, et al. Gas exchange kinetics in obese adolescents. Inferences on exercise tolerance and prescription. Am J Physiol Regul Integr Comp Physiol 2010; 299 (5): R1298-305.

40. Sietsema KE. Oxygen uptake kinetics in response to exercise in patients with pulmonary vascular disease. Am Rev Respir Dis 1992; 145 (5): 1052-7.

41. Regensteiner JG, Bauer TA, Reusch JE, Brandenburg SL, Sippel JM, Vogelsong AM, et al. Abnormal oxygen uptake kinetic responses in women with type II diabetes mellitus. J Appl Physiol (Bethesda, Md: 1985). 1998; 85 (1): 310-7.

42. Simoneau JA, Kelley DE. Altered glycolytic and oxidative capacities of skeletal muscle contribute to insulin resistance in NIDDM. J Appl Physiol (Bethesda, Md: 1985). 1997; 83 (1): 166-71.

43. Blair SN, Kohl HW, 3rd, Paffenbarger RS, Jr., Clark DG, Cooper KH, Gibbons LW. Physical fitness and all-cause mortality. A prospective study of healthy men and women. JAMA 1989; 262 (17): 2395-401.

44. Hopkins WG, Marshall SW, Batterham AM, Hanin J. Progressive statistics for studies in sports medicine and exercise science. Med Sci Sports Exerc 2009; 41 (1): 3-13. 